
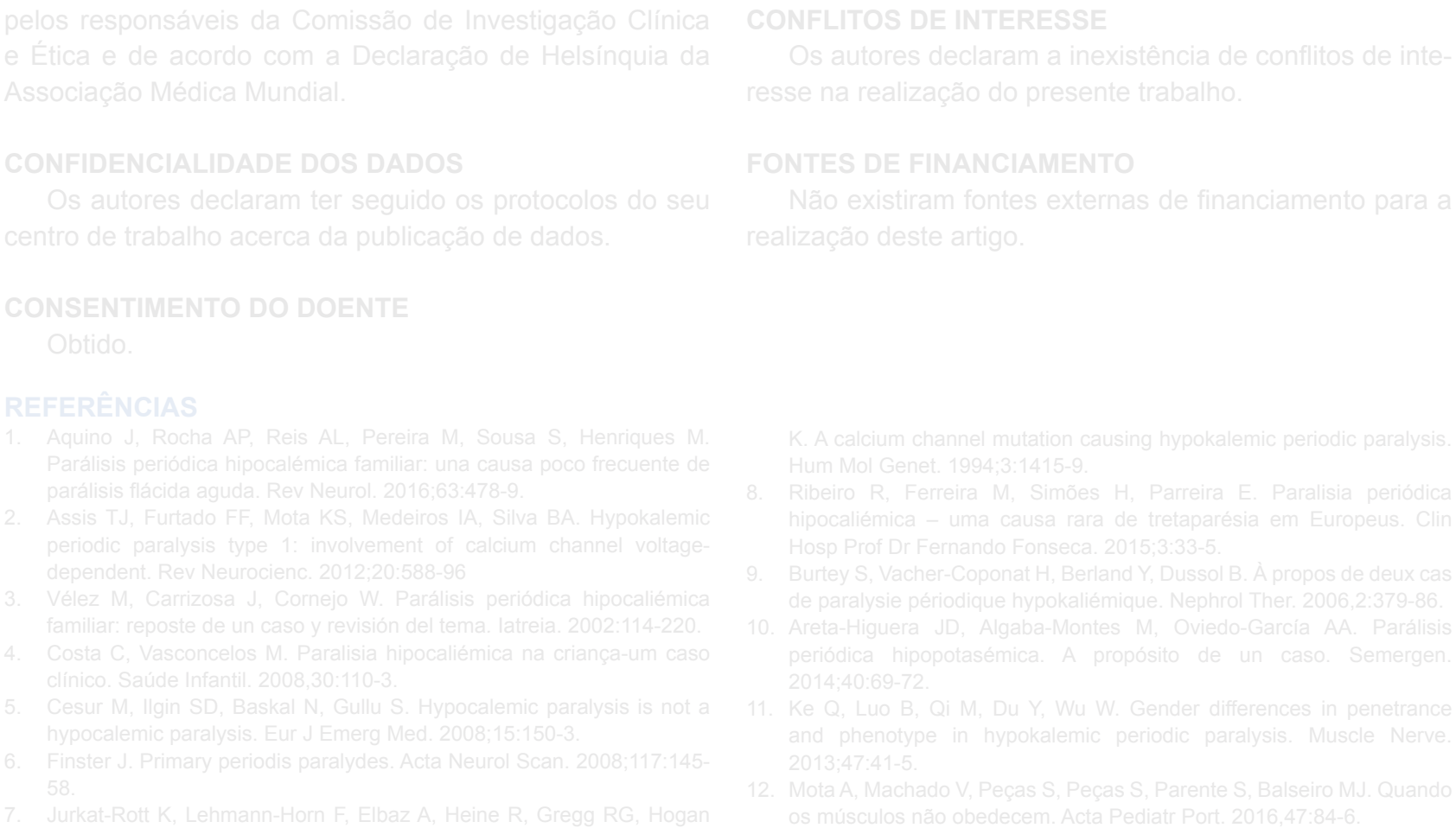

\title{
Coreia Aguda Generalizada, Distonia e Calcificações Cerebrais: A Propósito de um Caso Clínico
}

\section{Acute Generalized Chorea, Dystonia and Brain Calcifications: A Case Report}

\begin{abstract}
Joana RAMOS-LOPES $\square^{1}$, Ana BRÁS ${ }^{1}$, Ana MORGADINHO ${ }^{1}$, Fradique MOREIRA ${ }^{1}$
Acta Med Port 2019 May;32(5):402-406 - https://doi.org/10.20344/amp.11026

\section{RESUMO}

A calcificação dos núcleos da base, ou síndrome de Fahr, pode ser secundária a variadas doenças, nomeadamente as que cursam com envolvimento da paratiróide. Distúrbios do movimento são achados clínicos comuns, mas a coreia é observada em menos de $20 \%$ dos casos e a distonia apenas em $8 \%$. Apresentamos o caso de um homem de 49 anos com antecedentes de tiroidectomia, admitido no serviço de urgência com coreia aguda generalizada e distonia focal dolorosa dos pés, cujo estudo laboratorial revelava hipocalcémia e rabdomiólise e a tomografia computorizada crânio-encefálica mostrava calcificações parenquimatosas extensas com envolvimento dos núcleos da base. A alargada investigação complementar permitiu fazer o diagnóstico de síndrome de Fahr secundária a hipoparatiroidismo iatrogénico. Após estabilização da calcémia, a evolução clínica foi favorável com resolução dos sintomas neurológicos. A hipocalcémia deve ser investigada e corrigida depois de tiroidectomias, dada a irreversibilidade das calcificações intracerebrais e as potenciais consequências neurológicas e sistémicas.
\end{abstract}

Palavras-chave: Calcinose; Coreia; Distonia; Doenças dos Gânglios da Base; Hipocalcémia; Hipoparatiroidismo

\section{ABSTRACT}

Pathological basal ganglia calcification, or Fahr's Syndrome, can be secondary to a variety of diseases, namely parathyroid disturbances. Movement disorders are common clinical features, in which chorea is seen in less than $20 \%$ of cases and dystonia just in $8 \%$. We report the clinical case of a 49-year-old male with a history of thyroidectomy, who was admitted in Emergency Service with acute generalized chorea and focal painful feet dystonia. Laboratory analysis showed hypocalcemia and rhabdomyolysis, and computed tomography scan revealed parenchymal calcification with basal ganglia involvement. After complementary studies we established a Fahr's Syndrome diagnosis secondary to an iatrogenic hypoparathyroidism. Clinical management has been successful with stabilized calcium levels, with no more neurologic symptoms. Hypocalcemia should be readily investigated and treated after a thyroidectomy, given the irreversibility of intracerebral calcifications and potential neurological or systemic consequences.

Keywords: Basal Ganglia Diseases; Calcinosis; Chorea; Dystonia; Hypocalcemia; Hypoparathyroidism

1. Serviço de Neurologia. Centro Hospitalar e Universitário de Coimbra. Coimbra. Portugal.

$\bowtie$ Autor correspondente: Joana Ramos Lopes. joanaflopes6@gmail.com

Recebido: 07 de julho de 2018 - Aceite: 22 de agosto de 2018 | Copyright @ Ordem dos Médicos 2019 


\section{INTRODUÇÃO}

A calcificação dos núcleos da base é um achado comum, inespecífico, encontrado em $1 \%$ das tomografias computorizadas (TC) crânio-encefálicas (TC-ce). ${ }^{1}$ A síndrome de Fahr (SF), ou calcinose estriato-palido-dentada, é uma entidade neurodegenerativa, anatomo-clínica, associada a calcificações intracerebrais bilaterais e simétricas nos núcleos da base, tálamos, núcleos dentados, córtex e substância branca, com disfunção neurológica e/ou psiquiátrica progressiva. ${ }^{2-4}$ As manifestações clínicas incluem principalmente quadros frontais de alteração de comportamento, crises convulsivas e distúrbios do movimento, essencialmente parkinsonismo; a coreia é observada em menos de $20 \%$ dos casos e a distonia em apenas $8 \% .{ }^{5} \mathrm{~A}$ SF pode ser secundária a diversas doenças, desde vascular, tóxica, metabólica, infeciosa ou genética. Contudo, as principais são as disfunções endócrinas, nomeadamen- te o hipoparatiroidismo e os desequilíbrios fosfo-cálcio. ${ }^{6}$ O hipoparatiroidismo é a complicação mais frequente das intervenções cirúrgicas à tiróide ou paratiróides. Após tiroidectomia total, $20 \%$ - $30 \%$ poderão apresentar hipoparatiroidismo transitório e 1\% - 7\% permanente. ${ }^{7}$ É necessário seguimento clínico e laboratorial a longo prazo, ${ }^{2,8}$ já que níveis baixos de paratormona (PTH) e cálcio podem permanecer assintomáticos por períodos prolongados. ${ }^{2,9}$

\section{CASO CLÍNICO}

Homem, caucasiano, 49 anos, admitido no Serviço de Urgência (SU) após queda na via pública por movimentos involuntários hipercinéticos generalizados, de instalação aguda e agravamento em poucas horas. Sem história de viagens recentes, traumatismos, exposição a substâncias tóxicas ou a novos fármacos. O doente tinha sido
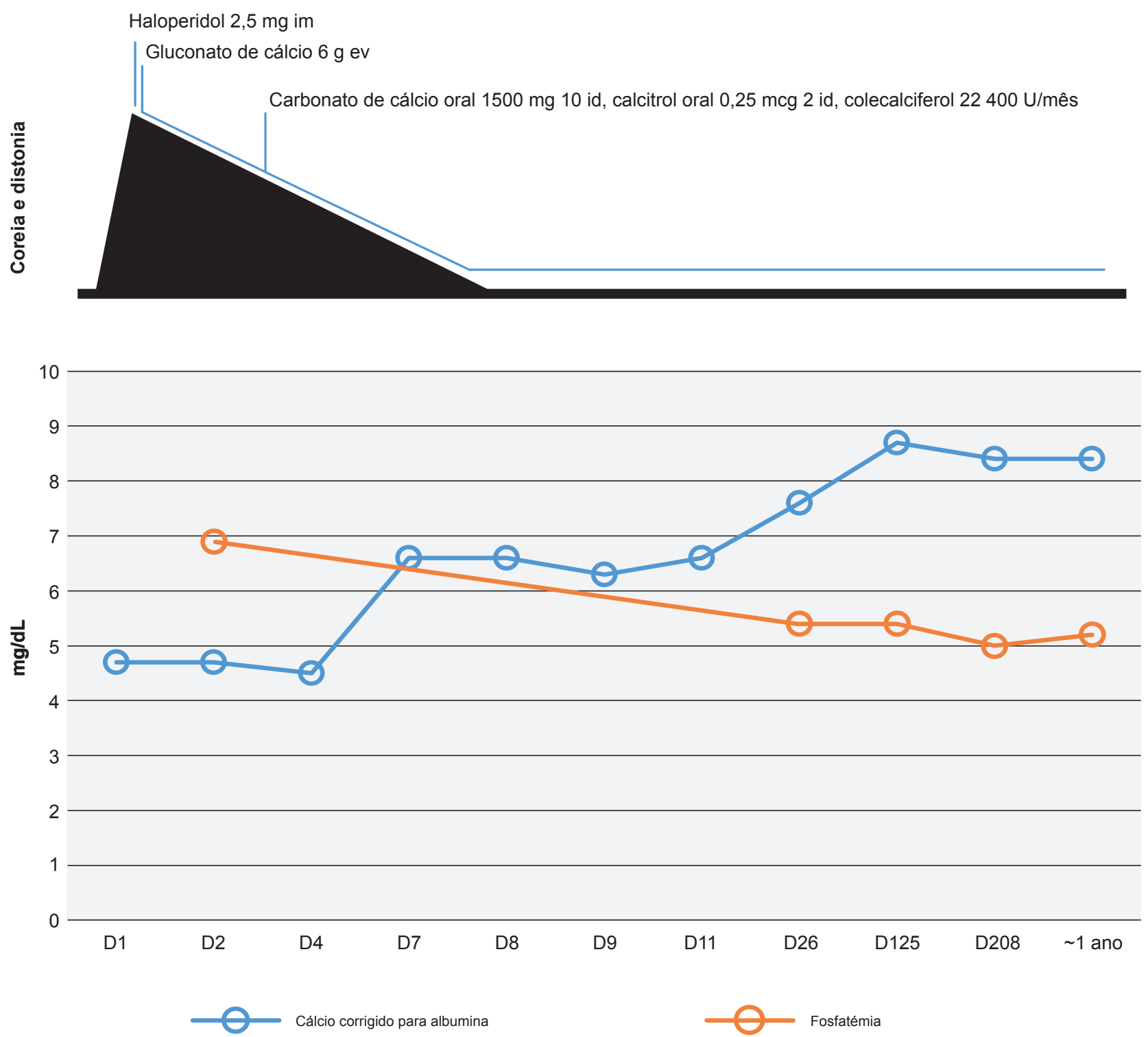

Figura 1 - Progressão dos níveis de cálcio corrigido para a albumina e fósforo, em relação com a clínica do doente e terapêuticas instituídas

Im: intramuscular; ev: endovenoso; id: por dia 
submetido a tiroidectomia há cerca de 40 anos por causa desconhecida, estando medicado com levotiroxina 0,1 $\mathrm{mg} / \mathrm{dia}$. Restantes antecedentes pessoais e familiares irrelevantes e sem história de episódios prévios. À admissão no SU estava consciente, orientado e ansioso; sinais vitais normais (temperatura auricular $37,0^{\circ} \mathrm{C}, \mathrm{FC} 97 \mathrm{bpm}$, TA 133/62 mmHg, saturação periférica de $\mathrm{O}_{2} 98 \%$ ). Na ava- liação neurológica apresentava disartria, decomposição dos movimentos oculares de perseguição, movimentos sacádicos lentos, impersistência ocular e na protusão da língua; coreia generalizada e distonia focal dolorosa dos pés, predomínio esquerdo; sem outros sinais de disfunção neurológica ou focais. A avaliação cognitiva breve era normal (Montreal Cognitive Assessment 26/30). Restante exame
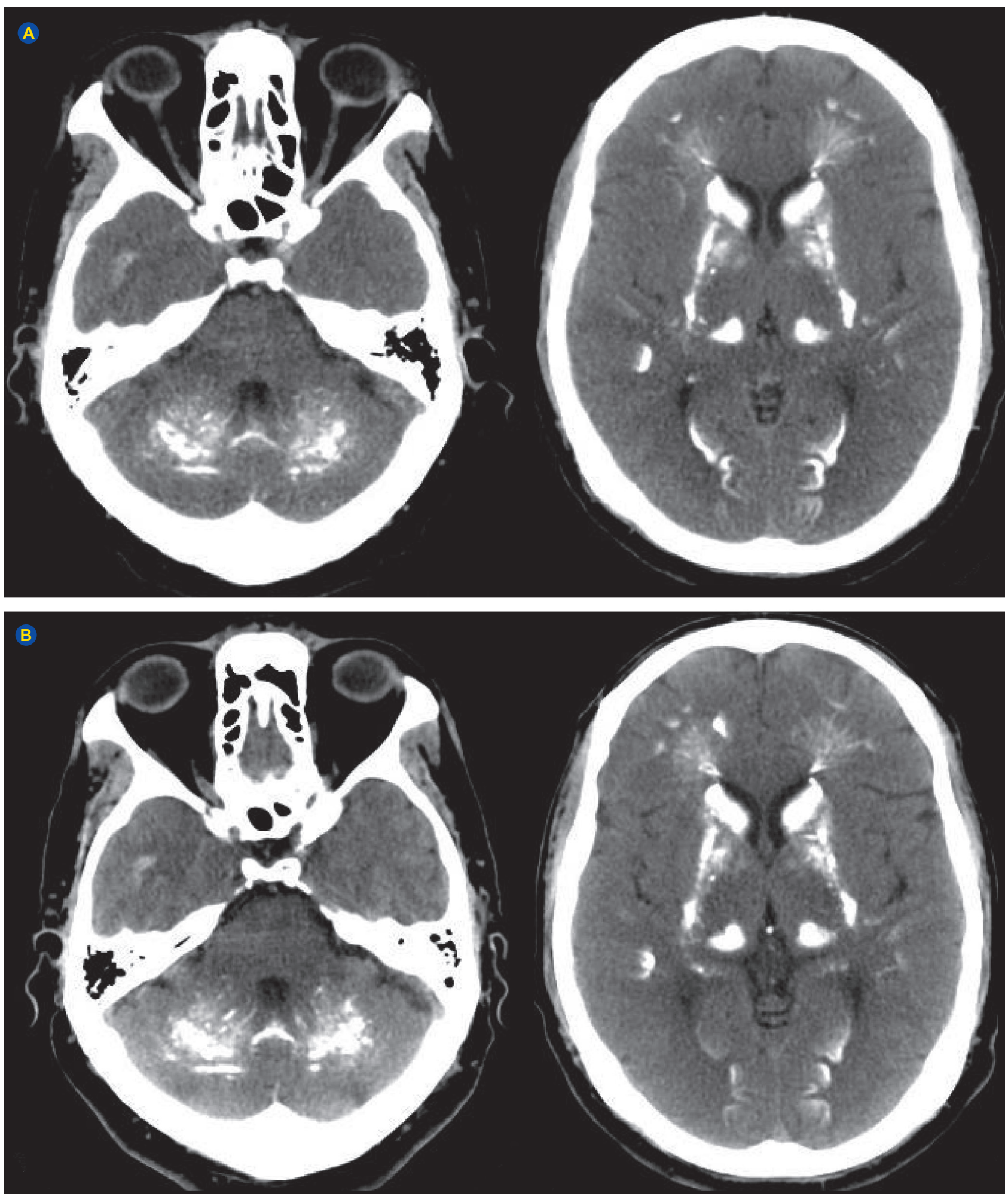

Figura 2 - (A) TC-ce inicial com calcificações bilaterais e simétricas envolvendo principalmente os núcleos da base, tálamos, córtex cerebelar e substância branca. (B) TC-ce após um ano, com padrão semelhante de calcificação, a nível de localização e extensão. 
físico geral sem alterações. O estudo analítico revelou nível de cálcio ajustado à albumina de $4,7(\mathrm{~N}: 8,8-10,6) \mathrm{mg} / \mathrm{dL}$ e creatina quinase $(\mathrm{CK})$ de $1096(\mathrm{~N}<171) \mathrm{U} / \mathrm{L}$. Hemograma, glicémia, funções renal e hepática, proteína $C$ reactiva e restante ionograma normais. Perante a hipocalcémia o doente realizou um electrocardiograma que não mostrou alterações. Para controlo sintomático da coreia generalizada administrou-se haloperidol $2,5 \mathrm{mg}$ intramuscular, com resposta favorável, e iniciou fluidoterapia e reposição endovenosa de cálcio. De forma a excluir lesão secundária, realizou TC-ce que revelou múltiplas e exuberantes calcificações bilaterais simétricas, de predomínio nos núcleos da base, tálamo e substância branca occipital. O doente foi internado para correção dos desequilíbrios electrolíticos e investigação complementar.

Considerando a apresentação aguda, a tiroidectomia prévia e os baixos níveis de cálcio, procedeu-se a estudo analítico alargado que evidenciou níveis de $\mathrm{PTH}<2,5$ (N: 9 - 72) pg/mL; hiperfosfatémia de 6,9 (N: 2,5 - 4,5) mg/dL; $25-\mathrm{OH}$-vitamina-D de $17(\mathrm{~N}>29) \mathrm{ng} / \mathrm{mL}$; e valores normais de magnésio, hormona tireoestimulante (TSH) e tiroxina 4 livre (T4I). A taxa de excreção de cálcio na urina/24 horas foi igualmente normal. O estudo ecográfico da tiróide conclui total ausência de paratiróides. A avaliação cardíaca com ecocardiograma transtorácico não mostrou alterações. Dada à presença de calcificações corticais cerebrais, o doente realizou um eletroencefalograma que não apresentou achados patológicos. Em colaboração com o Servi-

Tabela 1 - Diagnóstico diferencial de coreia aguda

\begin{tabular}{l}
\hline Etiologia \\
\hline Vascular \\
AVC isquémico/hemorrágico \\
Angioma cavernoso \\
MELAS (Mitochondrial encephalopathy with lactic acidosis and stroke) \\
Metabólica \\
Hiperglicémia sem cetoacidose \\
Hipoglicémia \\
Hipertiroidismo \\
Gravidez \\
Lesão ocupante de espaço \\
Inflamatória \\
Esclerose múltipla \\
Sarcoidose \\
Infecciosa \\
Toxoplasmose \\
Tuberculoma \\
VIH \\
Coreia de Sydenham (Streptococcus beta-hemolítico Grupo A) \\
Auto-imune/paraneoplásica \\
Lupus eritematoso sistémico \\
Síndrome do anticorpo anti-fosfolipídio \\
Encefalite por anticorpos anti-CRMP5/NMDA \\
Genética (Doença de Fahr) \\
latrogénica \\
Anti-epilépticos \\
Contraceptivos orais \\
Levodopa/agonistas dopaminérgicos \\
\hline
\end{tabular}

ço de Endocrinologia, manteve terapêutica de reposição de cálcio e vitamina $\mathrm{D}$, verificando-se uma melhoria progressiva da distonia dos pés e da coreia, com remissão total ao oitavo dia. Outras causas possíveis de calcificações cerebrais, como infeções (TORCH e VIH), doença vascular, outras causas metabólicas (ex: encefalopatias mitocondriais), exposição tóxica, radioterapia, doenças sistémicas (ex: lúpus eritematoso sistémico) e causas traumáticas, foram excluídas com base na história clínica e resultados de estudos complementares. Assim, foi feito o diagnóstico de SF secundária a hipoparatiroidismo iatrogénico. À data de alta, a calcémia estava estabilizada (Fig. 1), tendo sido prescrita terapêutica de suplementação com calcitriol, carbonato de cálcio e colecalciferol. Um ano após o internamento, a TC-ce de controlo revelou-se sobreponível, com o mesmo padrão de calcificações (Fig. 2). O doente mantém vigilância clínica e analítica em consultas de Neurologia e Endocrinologia, sem mais recorrência da coreia generalizada.

\section{DISCUSSÃO}

O caso clínico descrito ilustra o desafio diagnóstico da SF. Após a realização da TC-ce no SU, este tornou-se evidente, embora sem confirmação etiológica, dada a indisponibilidade imediata de confirmar o hipoparatiroidismo. A coreia aguda não é manifestação comum desta síndrome, sendo necessário fazer diagnóstico diferencial com outras entidades em contexto de urgência, principalmente com doença vascular cerebral que é a principal causa no adulto, ${ }^{10}$ de acordo com a história clínica e familiar, exame neurológico, testes laboratoriais e exames imagiológicos (Tabela 1). Contudo, neste caso, a sua identificação foi de extrema importância, pois o doente já apresentava rabdomiólise significativa, com risco de compromisso renal, e a hipocalcémia detectada exigia também uma correção rápida pelas potenciais consequências cardíacas.

O diagnóstico precoce da SF e o tratamento dirigido à causa subjacente podem prevenir a progressão da calcificação intracerebral e permitir um bom prognóstico, pelo que nos doentes submetidos a tiroidectomia total é imperativo a vigilância clínica e laboratorial dos desequilíbrios fosfo-cálcio. O mecanismo patogénico primário da calcinose cerebral condicionado pelo hipoparatiroidismo ainda não está totalmente esclarecido., ${ }^{4,6,11-14}$ Tem sido sugerido que as alterações funcionais resultam da disrupção dos circuitos núcleos da base-tálamo-córtex e/ou são dependentes da localização dos depósitos de cálcio. Pensa-se que a perda preferencial da projeção de neurónios $D_{2}$ é a base fisiopatológica para a coreia nestes doentes. ${ }^{14}$

Concluindo, importa ressalvar alguns pontos-chave do caso apresentado: (1) Um ano após o episódio o padrão de calcificação na TC-ce manteve-se sobreponível; este facto provavelmente explica que a presença inicial de coreia estava diretamente relacionada com a hipocalcémia e sua associação a hiperexcitabilidade muscular e não à presença das calcificações per se. (2) A correção da calcémia foi suficiente para a melhoria clínica; em normocalcémia o doente encontra-se assintomático. (3) Na presença de 
hipoparatiroisdismo, os níveis de cálcio são mais relevantes para a clínica do que os níveis de PTH. (4) A calcificação intracerebral é um processo irreversível, mesmo após estabilização do cálcio; não há tratamento específico à luz dos conhecimentos atuais.

\section{AGRADECIMENTOS}

Os autores gostariam de agradecer a Luís Cardoso, do Serviço de Endocrinologia do Centro Hospitalar e Universitário de Coimbra, pelo seu contributo essencial no diagnóstico, orientação e acompanhamento do doente, assim como a Luís Rito, do Serviço de Imagiologia/Neurorradiologia do Centro Hospitalar e Universitário de Coimbra, pelo auxílio na selecção das imagens.

\section{PROTECÇÃO DE PESSOAS E ANIMAIS}

Os autores declaram que os procedimentos seguidos estavam de acordo com os regulamentos estabelecidos

\section{REFERÊNCIAS}

1. Wong EM, Dahl M. Basal ganglia calcification in idiopathic hypoparathyroidism. BCMJ. 2013;55: 462-5.

2. Petrarca M, Scipioni R, Di Giosia P, Giorgini P, Ferri C, et al. A case of brain calcifications in postsurgical hypoparathyroidism. Intern Emerg Med. 2017;12:113-5.

3. Nagaraj A, Nair RP, Ganapathy S, Lakshman IK. Bilateral basal ganglia calcification secondary to FAHRs syndrome: a rare entity. J Neurol Disord. 2015;3:251.

4. Saleem S, Aslam HM, Anwar M, Anwar S, Saleem M, Saleem A, et al. Fahr's syndrome: literature review of current evidence. Orphanet J Rare Dis. 2013;8:156.

5. Manyam BV, Walters AS, Narla KR. Bilateral striopallidodentate calcinosis: clinical characteristics of patients seen in a registry. Mov Disord. 2001;16:258-64.

6. Jiménez-Ruiz A, Cárdenas-Sáenz O, Ruiz-Sandoval JL. Symmetrical and bilateral basal ganglia calcification. Case series and literature review. Gac Med Mex. 2018;154:258-62.

7. Tsai DR, Huang SH, Lin SH. Bilateral striopallidodentate calcinosis pelos responsáveis da Comissão de Investigação Clínica e Ética e de acordo com a Declaração de Helsínquia da Associação Médica Mundial.

\section{CONFIDENCIALIDADE DOS DADOS}

Os autores declaram ter seguido os protocolos do seu centro de trabalho acerca da publicação de dados.

\section{CONSENTIMENTO DO DOENTE}

Obtido.

\section{CONFLITOS DE INTERESSE}

Os autores declaram não terem qualquer conflito de interesse relativamente ao presente artigo.

\section{FONTES DE FINANCIAMENTO}

Os autores declaram não ter recebido subsídios ou bolsas para a elaboração do artigo.

secondary to postsurgical hypoparathyroidism. BMJ Case Rep. 2013;2013.

8. Shoback DM, Bilezikian JP, Costa AG, Dempster D, Dralle H, Khan $A A$, et al. Presentation of hypoparathyroidism: etiologies and clinical features. J Clin Endocrinol Metab. 2016;101:2300-12.

9. Khan MI, Waguespack SG, Hu MI. Medical management of postsurgical hypoparathyroidism. Endocr Pract. 2011;17:S18-25.

10. Cardoso F, Seppi K, Mair KJ, Wenning GK, Poewe W. Seminar on choreas. Lancet Neurol. 2006;5:589-602.

11. Munhoz RP, Scorr LM, Factor SA. Movement disorders emergencies. Curr Opin Neurol. 2015;28:406-12.

12. Dos Santos VM, Da Mata AM, Ribeiro KR, Calvo IC. Fahr's syndrome and secondary hypoparathyroidism. Rom J Intern Med. 2016;54:63-5.

13. Deng $\mathrm{H}$, Zheng W, Jankovic J. Genetics and molecular biology of brain calcification. Ageing Res Rev. 2015;22:20-38.

14. Kono S, Manabe Y, Tanaka T, Fujii D, Sakai Y, Narai H, et al. A case of Fahr's disease presenting as chorea successfully treated by the use of quetiapine. Clin Med Case Rep. 2009;2:63-5. 